\title{
A Face-Split Study to Evaluate the Effects of Microneedle Radiofrequency with Q-Switched Nd:YAG Laser for the Treatment of Melasma
}

\author{
Jin Woong Jung, Won Oh Kim, Hae Ra Jung ${ }^{1}$, Sung Ae Kim, Young Wook Ryoo \\ Departments of Dermatology and ${ }^{1}$ Pathology, Keimyung University School of Medicine, Daegu, Korea
}

Background: Laser toning using a low-fluence 1,064 nm Q-switched Nd:YAG laser is one of the most frequently used treatment modalities for melasma. However, this therapy is time consuming because it requires a lot of treatment sessions. Recently, it has been reported that transdermal radiofrequency (RF) is effective for the treatment of melasma. Objective: To determine whether microneedle RF conduction could be an adjunct therapy for melasma, we have studied the effect of simultaneous treatments with laser toning and RF for melasma. Methods: Fifteen patients with melasma underwent five sessions of laser toning and microneedle RF on the right side of the face, and only laser toning on the left side. Responses to treatments were evaluated using the Mexameter $^{\mathbb{R}}$ (Courage Khazaka, Germany) score, the pigmentation and severity index (PSI) score, and the patient's overall assessment. Additionally, an electron microscopic study of a skin biopsy was performed. Results: Both laser toning and combination therapy showed significant decreases in the Mexameter ${ }^{\mathbb{R}}$ and PSI score after five treatment sessions. Combination therapy showed a more significant improvement of melasma than laser toning. No remarkable side effects were reported. Electron microscopic analysis showed

Received March 27, 2018, Revised May 26, 2018, Accepted for publication August 30, 2018

Corresponding author: Young Wook Ryoo, Department of Dermatology, Keimyung University Dongsan Medical Center, 56 Dalseong-ro, Jung-gu, Daegu 41931, Korea. Tel: 82-53-250-7624, Fax: 82-53-250-7626, E-mail: ryoo111@dsmc.or.kr

ORCID: https://orcid.org/0000-0002-2477-6263

This is an Open Access article distributed under the terms of the Creative Commons Attribution Non-Commercial License (http://creativecommons. org/licenses/by-nc/4.0) which permits unrestricted non-commercial use, distribution, and reproduction in any medium, provided the original work is properly cited.

Copyright $\odot$ The Korean Dermatological Association and The Korean Society for Investigative Dermatology a greater number of vacuolar changes and increased loosening of melanocytes and adjacent epidermal cells after combination therapy. Conclusion: The combination treatment of laser toning and microneedle RF therapy showed a better therapeutic effect for melasma than laser toning alone. Therefore, the microneedle RF technique could be a new and safe adjunct therapy for the treatment of melasma. (Ann Dermatol 31(2) 133 138, 2019)

-Keywords-

Lasers, Melanosis, Radiofrequency therapy

\section{INTRODUCTION}

Melasma is a common pigmentary disorder that is prevalent in middle-aged women ${ }^{1}$. It is caused by disruption of homeostasis in dermal pigmentation. Ultraviolet (UV) irradiation, female sex hormones, inflammatory processes, and microenvironments of the dermal structure combined with genetic factors are considered important elements in the pathogenesis of melasma ${ }^{2}$. Treatment of melasma is difficult due to its chronic and relapsing nature. First-line therapy for melasma includes broad-spectrum sunscreens and topical hypopigmentation agents. Second-line therapy includes chemical peeling agents, and third-line therapy includes laser modalities ${ }^{3}$. Diverse laser therapies including intense pulsed light, Q-switched lasers, picosecond lasers, nonablative fractionated lasers, and ablative fractionated resurfacing lasers have been studied in many clinical trials to evaluate the efficacies and side effects of the treatment of melasma ${ }^{3}$. Laser therapies have become common treatment options for patients with refractory cases; however, they can have unpredictable results, such as post 
inflammatory hyperpigmentation and thermal damage $\mathrm{e}^{3,4}$ The low-fluence 1,064 nm Q-switched Nd:YAG (QSNY) laser has become a widely used modality recently because of its minimal downtime and low risk of postinflammatory hyperpigmentation $^{3}$. It is thought to selectively target the cytoplasmic melanin granules without causing cellular destruction, so called 'subcellular selective thermolysis.' Though the low-fluence QSNY is an effective and safe treatment option for melasma, it requires many treatment sessions to obtain a satisfying result and the high recurrence rate is still a problem ${ }^{5}$. Therefore, finding a new treatment modality that can reinforce the effect of the low-fluence QSNY laser without serious side effects is necessary.

Microneedle radiofrequency (RF) transmits RF currents from the needle tips, which induces direct changes in the dermoepidermal junction and dermis with minimal ablation of the epidermis ${ }^{6}$. It has been widely used for skin rejuvenation with a good safety profile and minimal downtime. Recently, there have been a few case reports concerning the effect of microneedle RF on melasma ${ }^{7}$.

Microneedle RF could be effective in the treatment of melasma because it can improve the impaired extracellular matrix (ECM) of skin, which is commonly found in a melasma lesion ${ }^{6}$. It is also expected that through the microneedling effect, transepidermal elimination of melanin and drug delivery through the skin barrier can be increased ${ }^{8,9}$. Therefore, the combination of microneedle RF and QSNY could represent a new melasma treatment regimen with a synergistic effect and good safety profile. This study aimed to evaluate the efficacy and safety of combination treatment with microneedle RF and QSNY laser toning for melasma, as compared with conventional QSNY treatment alone.

\section{MATERIALS AND METHODS}

Fifteen Korean patients (14 women and one man) who were clinically diagnosed with melasma were enrolled in the study. Patients who were pregnant or taking oral contraceptive were excluded. Patients with a history of receiving laser treatment on the face for any reason within 1 year were also excluded. Patients with comorbid diseases that can trigger hyperpigmentation, such as hypertension, hyperlipidemia, chronic inflammatory skin disease, and psychiatric disease, were excluded ${ }^{10}$. Patients were instructed not to use other forms of treatments for melasma except the therapy applied in this research. The study was approved by the medical ethics and human research committees of Keimyung University Dongsan Medical Center (Daegu, Korea; IRB no. 2016-09-035). Informed consent was obtained from each patient before enrollment. We received the patient's consent form about publishing all photographic materials.

All patients underwent five treatment sessions of microneedle RF (Secret ${ }^{\circledR}$; Ilooda Inc., Suwon, Korea) and the low-fluence $1,064 \mathrm{~nm}$ QSNY laser (Curas ${ }^{\circledR}$; Ilooda Inc.) on the right side of face, and only the low-fluence 1,064 nm QSNY laser on the left side, with a 2-week interval between treatment sessions. Before the treatment, $2.5 \%$ lidocaine and $2.5 \%$ prilocaine cream $\left(\right.$ Emla $^{\circledR}$; AstraZeneca Pharmaceuticals LP, Wilmington, DW, USA) were applied to the lesions. Patients received treatment with the low-fluence 1,064 nm QSNY laser (PTP mode, fluence $1.19 \mathrm{~J} / \mathrm{cm}^{2}$, spot size $8 \mathrm{~mm}$, median shot 2,350.1 \pm 524.80 ) on both sides of face to reach the end point of mild erythema. Then, the fractional microneedle RF (intensity $50 \%$, depth $1 \mathrm{~mm}$, pulse duration $50 \mathrm{~ms}$, single pass) was performed on the right side of the face on the same day. This device is a minimally invasive microneedle RF system, which has $25(5 \times 5$ parallel rows) non-insulated bipolar electrodes. Patients were instructed to always apply broad-spectrum sunscreen on the entire face when outdoors.

Responses to treatments were evaluated using the Mexameter $^{\circledR}$ (Courage Khazaka, Cologne, Germany), the pigmentation and severity index (PSI) score, and the patient's overall assessment. The Mexameter ${ }^{\mathbb{R}}$ can measure the melanin index and erythema index by detecting lights reflected by the skin ${ }^{11}$.

In order to minimize error caused by the measuring position of the Mexameter ${ }^{\circledR}$, photographs were taken at a baseline visit and three points were marked on the most conspicuous sites of melasma. Thereafter, measurements were made at the same position with reference to the baseline photograph. Standardized digital photographs were taken at every visit under the same lighting condition (EOS $650 D^{\mathbb{R}}$; Canon, Tokyo, Japan). Two different physicians recorded the PSI score at baseline (pretreatment), on the third visit (before session 3), and on the sixth visit (2 weeks after session 5). The PSI score is accessed by quantifying changes in pigmentation, as previously described ${ }^{12}$. At the end of the study, patients completed a questionnaire to assess their satisfaction with each treatment as follows: unsatisfied or worse, no change, mild improvement, moderate improvement, and significant improvement. A numeric pain intensity scale $(0 \sim 10)$ and any side effects were also recorded by the patients.

Statistical analyses were performed using IBM SPSS Statistics ver. 24.0 (IBM Co., Armonk, NY, USA). Wilcoxon signed rank test was used to analyze the Mexameter ${ }^{\circledR}$ score, PSI score, patient overall assessment, and pain score. We considered $p$-values $<0.05$ to be statistically significant. 
Additionally, an electron microscopy study of a 2-mm punch biopsy specimen was performed after completion of all sessions in nine of the 15 patients who agreed to undergo biopsy.

\section{RESULTS}

All 15 patients (one man and 14 women, mean age $43.1 \pm$ 5.88 years, two Fitzpatrick skin type III and 13 Fitzpatrick skin type IV, duration of melasma $10.67 \pm 4.91$ years) completed the five sessions of treatment. There was no significant difference in the baseline Mexameter ${ }^{(\mathbb{R}}$ and PSI scores between the two treatment modalities (Table 1).

\section{Objective assessment of melasma improvement}

Compared to baseline, both the combination treatment and laser toning treatment showed a significant decrease in the Mexameter ${ }^{\circledR}$ and PSI scores after five treatment sessions. On the third visit, the Mexameter ${ }^{\mathbb{R}}$ score of the combination treatment was more significantly decreased than the laser toning treatment. On the sixth visit (2 weeks after session 5), both the Mexameter ${ }^{\mathbb{R}}$ and PSI scores of the combination treatment were more significantly decreased than those of the laser toning treatment. These results demonstrate that a greater clinical improvement of melasma was observed after the combination therapy (Table 1). Representative photographs of each facial side after treatment are shown in Fig. 1.
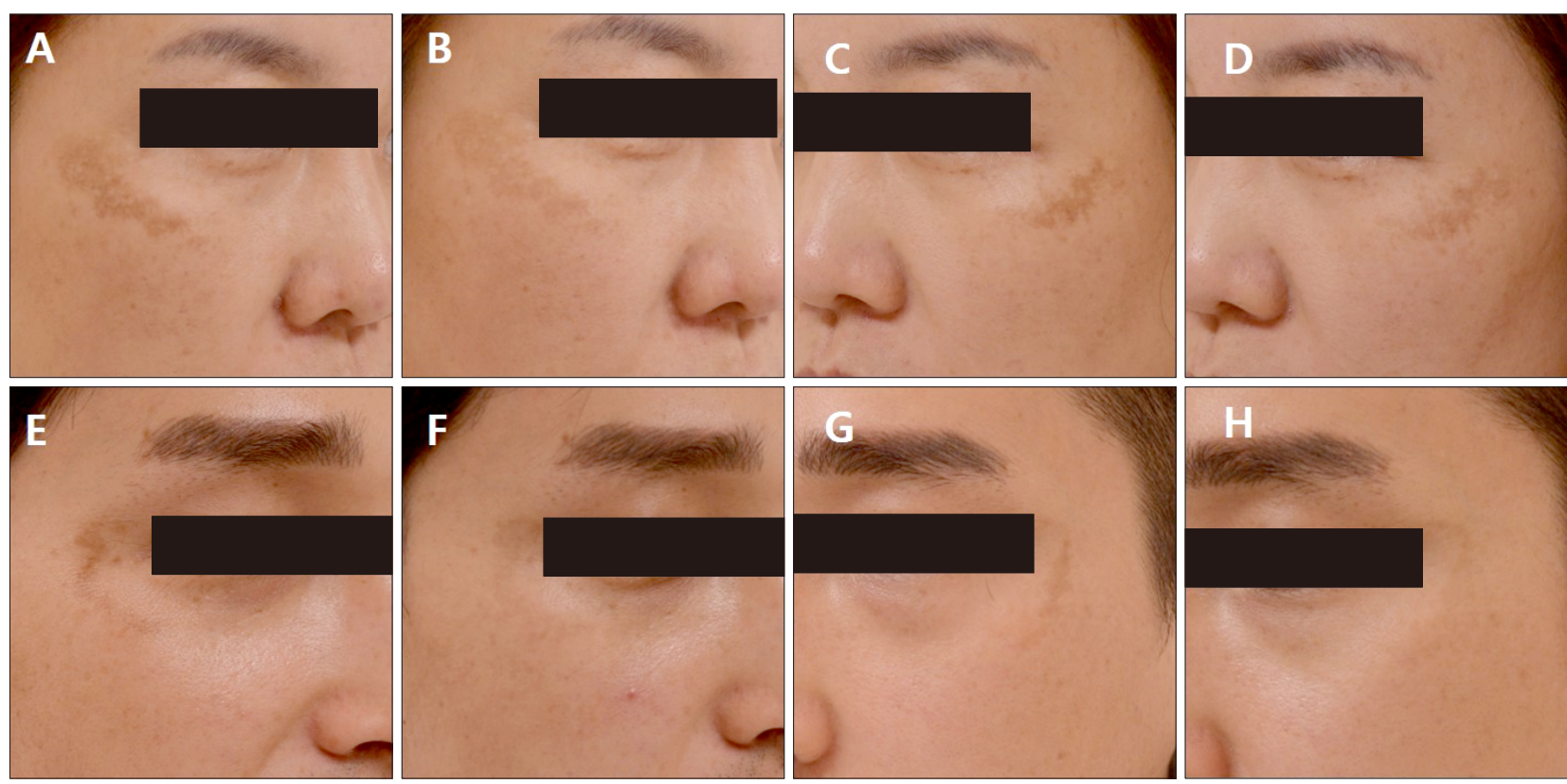

\section{Patients' overall assessment and side effects}

The patients' mean satisfaction score was $3.53 \pm 0.81$ after combination therapy and $3.13 \pm 0.96$ after laser toning therapy (Table 2). The proportion of patients who assessed their satisfaction as a 'moderate improvement' or 'significant improvement' was $46.7 \%$ (seven out of 15 ) for the combination treatment and $26.7 \%$ (four out of 15 ) for the laser toning treatment. The mean pain score was higher during

Table 1. Treatment results

\begin{tabular}{|c|c|c|c|c|}
\hline & Baseline & Visit 3 & Visit 6 & $p$-value \\
\hline \multicolumn{5}{|c|}{ Mexameter ${ }^{R}$} \\
\hline $\begin{array}{l}\mathrm{RF}+ \\
\text { toning }\end{array}$ & $148.98 \pm 57.45$ & $\begin{array}{c}96.30 \pm 26.85 \\
(-35.8 \%)\end{array}$ & $\begin{array}{c}65.55 \pm 26.24 \\
(-53.0 \%)\end{array}$ & 0.000 \\
\hline Toning & $143.24 \pm 54.85$ & $\begin{array}{c}114.23 \pm 43.49 \\
(-20.3 \%)\end{array}$ & $\begin{array}{c}87.31 \pm 42.13 \\
(-39.2 \%)\end{array}$ & 0.006 \\
\hline$p$-value ${ }^{\dagger}$ & 0.460 & $0.041^{*}$ & $0.005^{*}$ & \\
\hline \multicolumn{5}{|l|}{ PSI score } \\
\hline $\begin{array}{l}\mathrm{RF}+ \\
\text { toning }\end{array}$ & $10.20 \pm 3.06$ & $\begin{array}{c}7.90 \pm 2.70 \\
(-22.5 \%)\end{array}$ & $\begin{array}{l}5.50 \pm 2.05 \\
(-46.1 \%)\end{array}$ & 0.000 \\
\hline Toning & $11.13 \pm 4.88$ & $\begin{array}{c}9.00 \pm 4.19 \\
(-19.1 \%)\end{array}$ & $\begin{array}{c}7.63 \pm 3.16 \\
(-31.4 \%)\end{array}$ & 0.037 \\
\hline$p$-value ${ }^{\dagger}$ & 0.241 & 0.066 & $0.002 *$ & \\
\hline
\end{tabular}

Values are presented as mean \pm SD or mean \pm SD (percentage change). RF: radiofrequency, PSI: pigmentation area and severity index, SD: standard deviation. ${ }^{*} p<0.05 .{ }^{\dagger}$ Difference between sides treated with combination therapy and laser toning.

Fig. 1. (A, C) A 44-year-old woman with skin type IV at baseline, and (B, D) 2 weeks after five treatment sessions. (E, G) A 35-year-old man with skin type IV at baseline, and $(\mathrm{F}, \mathrm{H}) 2$ weeks after five treatment sessions. $(\mathrm{A}, \mathrm{B}, \mathrm{E}, \mathrm{F})$ Combination therapy-treated side of face. (C, D, G, H) Laser toning-treated side of face. 
JW Jung, et al

combination therapy $(3.40 \pm 0.71)$ than laser toning $(1.73 \pm 0.57)$. Pain during therapy was well tolerated by all patients. No significant side effects were reported.

\section{Electron microscopic analysis}

Transmission electron microscopic images demonstrated shrinkage of melanocyte cytoplasm after both treatment modalities. Loosening or slit-like spaces between the melanocytes and adjacent epidermal cells were more evident after combination therapy (Fig. 2A D ). The mitochondria

Table 2. Patients' overall assessment

\begin{tabular}{lccc}
\hline & RF + toning & Toning & $p$-value \\
\hline Patient satisfaction (score) & & & \\
Unsatisfied or worse (1) & 0 & 0 & \\
No change (2) & 1 & 1 & \\
Mild improvement (3) & 7 & 10 & \\
Moderate improvement (4) & 5 & 2 & \\
Significant improvement (5) & 2 & 2 & \\
Average & $3.53 \pm 0.81$ & $3.13 \pm 0.96$ & 0.107 \\
Pain scale & $3.40 \pm 0.71$ & $1.73 \pm 0.57$ & 0.001 \\
Side effects & 0 & 0 & \\
\hline
\end{tabular}

Values are presented as number or mean \pm standard deviation. RF: radiofrequency. were more edematous and the number of micro-vesicles was also greater after combination therapy (Fig. 2E, F).

\section{DISCUSSION}

The RF device was first approved for facial wrinkle reduction in 2002 by the U.S. Food and Drug Administration ${ }^{6}$. There are two different types of RF current delivery: a monopolar mode that utilizes an active electrode and a dispersive electrode, and a bipolar mode that consists of two active electrodes ${ }^{6}$. RF has been used for many indications, such as skin tightening, wrinkle reduction, and the treatment of acne scars and cellulite ${ }^{8}$. It emits an electrical current into the skin, and thermal energy is produced when the current enters the tissue (which serves as impedance). The thermal energy contracts old collagen fibers and stimulates dermal fibroblasts to synthesize new collagen. Unlike other laser lights, RF energy does not interfere with tissue distraction or chromophore absorption. Therefore, it can provide a more controlled depth of energy penetration and be widely used in all skin types ${ }^{8}$.

Microneedle RF is a relatively recently developed method. The microneedle RF tip consists of parallel rows of microneedles arranged in a bipolar array. RF energy flows between microneedles, which have positive or negative po-
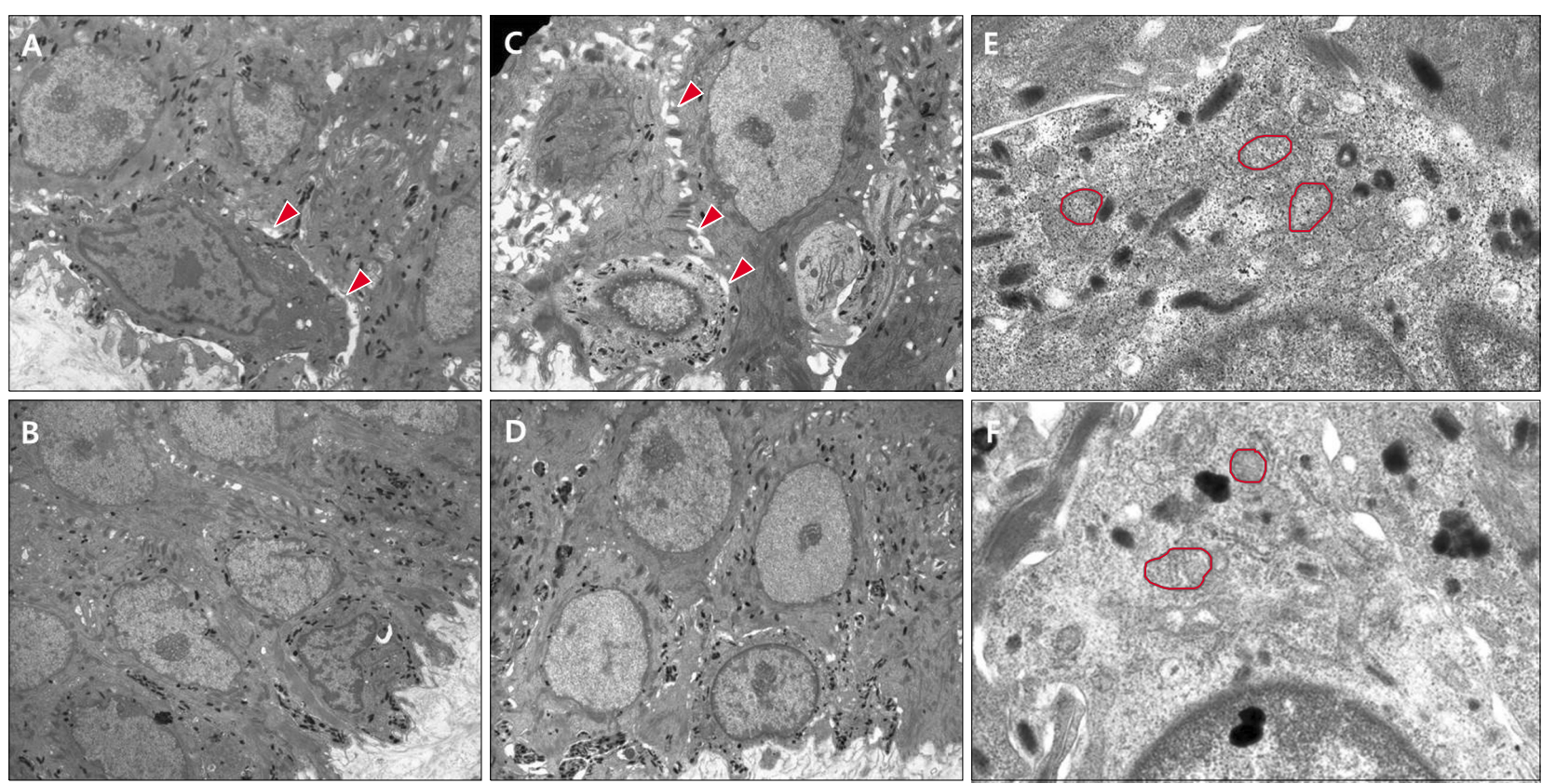

Fig. 2. (A $\sim \mathrm{D})$ Transmission electron microscope images of the epidermis layer $(\times 3,000)$. (A) and (C) are images of the epidermis after combination therapy. (B) and (D) are images of the epidermis after toning therapy. Red arrowheads show loosening of melanocytes and adjacent epidermal cells. Images (A) and (C) show increased loosening of melanocytes and adjacent epidermal cells. Shrinkage of the melanocyte cytoplasm was found after both treatment modalities. (E) and (F) are images of micro-organelles in the melanocytes after both treatment modalities. The mitochondrias (red circles) were more edematous and the number of micro-vesicles was greater after combination therapy $(E)$ than toning alone $(F)(\times 6,000)$. 
larity, so that each pair forms a powerful closed circuit bipolar RF current ${ }^{6}$. The tips can be insulated or non-insulated; with non-insulated tips, the entire needle serves as the electrode, whereas the energy flows only through the tip of the needle with insulated tips ${ }^{6}$. Theoretically, insulated tips can deliver RF to the dermis without damaging the epidermis. However, due to differences in the impedance between the epidermis (high impedance) and the dermis (low impedance), a water drop-shaped zone of electrothermal coagulation in the dermis is formed ${ }^{12}$. Therefore, the use of non-insulated tips is also safe and can be used without side effects ${ }^{12,13}$. Moreover, Harth and Frank $^{13}$ reported that non-insulated needles allow better coagulation and heating of higher volumes of dermal tissue as compared to insulated needles. Microneedling itself has been used for pigment disorders in combination with topical drugs. It increases the transepidermal administration of whitening agents and transepidermal elimination of melanin ${ }^{9}$.

Choi et al. ${ }^{7}$ first reported five cases of refractory melasma successfully treated using an invasive micro-pulsed electrical signaling device. Kim et al. ${ }^{14}$ reported a significant improvement in the melanin index and erythema index after the application of RF. Based on these reports, our study was conducted on the concept that combination therapy of microneedle RF and QSNY would be more effective in the treatment of melasma than the traditional laser toning therapy. As a result, combination therapy showed a greater clinical improvement of melasma than the laser toning therapy alone. We hypothesize that the mechanism behind RF treatment of melasma might be related to the effect of RF on the dermal ECM conditions.

Three main characteristics of the dermal condition in melasma patients include impairment of the dermal ECM, increased vascularity, and the increased activity of mast cells $^{15,16}$. Abnormalities of the dermal ECM are most commonly observed. Moderate to severe solar elastosis was reported in over $90 \%$ of patients with melasma. This suggests that a decrease in the ECM components caused by photoaging plays an important role in the development of melasma. Microneedle RF could reverse solar elastosis, which may reduce pro-melanogenesis paracrine factors and melanogenesis ${ }^{7}$.

Park et al. ${ }^{17}$ reported the therapeutic effect of microneedle RF in rosacea patients, the mechanism of which is due to an anti-inflammatory and anti-angiogenetic effect. UV irradiation up-regulates the release of histamine from dermal mast cells, which stimulates the proliferation and migration of melanocytes ${ }^{16,17}$. After RF treatment, the markers related to angiogenesis (vascular endothelial growth factor) and inflammation nuclear factor $\kappa \mathrm{B}$ and interleukin-8) were significantly decreased and the average mast cell count was also decreased compared to baseline. These findings demonstrate that microneedle RF may reduce angiogenesis, inflammation, and mast cell activation, which also play an important causal role in melasma. Therefore, we believe that the same mechanisms would lead to an improvement of melasma by RF treatment in the current study.

Mun et al. ${ }^{18}$ reported electron microscopic findings of melasma after low-fluence QSNY laser therapy including decreased dendrites, shrinkage of the cytoplasm in melanocytes, and a decreased number of stage IV melanosomes. Our electron microscopic study also showed shrinkage of the melanocyte cytoplasm in both treatment modalities. The mitochondria were more edematous and the number of micro-vesicles was more prominent after combination therapy. The shrinkage and vacuolar changes of the cytoplasm indicate melanocyte damage; therefore, the production of melanin in damaged melanocytes is expected to be reduced. Compared to laser toning therapy, a more severe loosening between melanocytes and adjacent epidermal cells was found after combination therapy. Transdermal elimination of melanin could be increased through these loosened spaces. These findings suggest grounds for the clinical benefit of RF and laser toning combination therapy. Considering the thickness of the epidermis, the tip of the microneedle RF could reach under the basement membrane zone, thus, creating new channels to the dermal layer ${ }^{19}$. We hypothesize that these pores allowed the melanosomes to drop off to the dermis.

This study proved the safety and efficacy of combination therapy with microneedle RF and low-fluence 1,064 nm QSNY laser for the treatment of melasma. The clinical improvement of melasma was more prominent after combination therapy, and patients also reported satisfaction with the additional benefits of improved periocular wrinkles on the side treated with combination therapy. No serious side effects of combination therapy were reported, although patients complained of more pain during the combination therapy. Therefore, more attention should be given to provide thorough local anesthesia for this regimen.

There are some limitations of the present study. First, all patients were of a similar ethnic background. Second, patients were observed for only up to 2 weeks after the five treatment sessions; therefore, examination of the long-term effects of combination therapy should be continued. In addition, adequate treatment parameters such as the intensity and depth of microneedle RF and its precise mechanism of action remain to be elucidated.

In conclusion, the combination therapy of microneedle RF and a low-fluence 1,064 nm QSNY laser is safe and shows 
an improved therapeutic effect for melasma compared to single low-fluence 1,064 nm QSNY laser treatment alone. Therefore, this regimen could represent a superior treatment option for melasma. Future research with a longer follow-up and determination of the adequate intensity and depth of RF treatment for melasma are needed.

\section{ACKNOWLEDGMENT}

This work was funded by the Ilooda Inc., Korea.

\section{CONFLICTS OF INTEREST}

The authors have nothing to disclose.

\section{ORCID}

Jin Woong Jung, https://orcid.org/0000-0001-7592-3035

Won Oh Kim, https://orcid.org/0000-0001-6826-6444

Hae Ra Jung, https://orcid.org/0000-0002-1477-6606

Sung Ae Kim, https://orcid.org/0000-0002-6040-6630

Young Wook Ryoo, https://orcid.org/0000-0002-2477-6263

\section{REFERENCES}

1. Lee AY. Recent progress in melasma pathogenesis. Pigment Cell Melanoma Res 2015;28:648-660.

2. Handel AC, Miot LD, Miot HA. Melasma: a clinical and epidemiological review. An Bras Dermatol 2014;89:771-782.

3. Trivedi MK, Yang FC, Cho BK. A review of laser and light therapy in melasma. Int J Womens Dermatol 2017;3:11-20.

4. Manaloto RM, Alster T. Erbium:YAG laser resurfacing for refractory melasma. Dermatol Surg 1999;25:121-123.

5. Gokalp H, Akkaya AD, Oram Y. Long-term results in lowfluence 1064-nm Q-Switched Nd:YAG laser for melasma: is it effective? J Cosmet Dermatol 2016;15:420-426.

6. Lolis MS, Goldberg DJ. Radiofrequency in cosmetic dermatology: a review. Dermatol Surg 2012;38:1765-1776.

7. Choi M, Choi SH, Kang JS, Cho SB. Successful treatment of refractory melasma using invasive micro-pulsed electric signal device. Med Laser 2015;4;39-44.

8. Krueger N, Sadick NS. New-generation radiofrequency technology. Cutis 2013;91:39-46.

9. Cohen BE, Elbuluk N. Microneedling in skin of color: a review of uses and efficacy. J Am Acad Dermatol 2016;74: 348-355.

10. Clinard V, Smith JD. Drug-induced skin disorder. US Pharm 2012;37:HS11-HS18.

11. Matias AR, Ferreira $M$, Costa $P$, Neto P. Skin colour, skin redness and melanin biometric measurements: comparison study between Antera $(R)$ 3D, Mexameter $(R)$ and Colorimeter(R). Skin Res Technol 2015;21:346-362.

12. Na J, Zheng Z, Dannaker C, Lee SE, Kang JS, Cho SB. Electromagnetic initiation and propagation of bipolar radiofrequency tissue reactions via invasive non-insulated microneedle electrodes. Sci Rep 2015;5:16735.

13. Harth Y, Frank I. In vivo histological evaluation of noninsulated microneedle radiofrequency applicator with novel fractionated pulse mode. J Drugs Dermatol 2013;12:14301433.

14. Kim JE, Lee HW, Kim JK, Moon SH, Ko JY, Lee MW, et al. Objective evaluation of the clinical efficacy of fractional radiofrequency treatment for acne scars and enlarged pores in Asian skin. Dermatol Surg 2014;40:988-995.

15. Kwon SH, Hwang YJ, Lee SK, Park KC. Heterogeneous pathology of melasma and its clinical implications. Int J Mol Sci 2016;17:E824.

16. Kang $\mathrm{WH}$, Yoon $\mathrm{KH}$, Lee $\mathrm{ES}$, Kim J, Lee $\mathrm{KB}$, Yim $\mathrm{H}$, et al. Melasma: histopathological characteristics in 56 Korean patients. Br J Dermatol 2002;146:228-237.

17. Park SY, Kwon HH, Yoon JY, Min S, Suh DH. Clinical and histologic effects of fractional microneedling radiofrequency treatment on rosacea. Dermatol Surg 2016;42:1362-1369.

18. Mun JY, Jeong SY, Kim JH, Han SS, Kim IH. A low fluence Q-switched Nd:YAG laser modifies the 3D structure of melanocyte and ultrastructure of melanosome by subcellular-selective photothermolysis. J Electron Microsc (Tokyo) 2011;60:11-18.

19. Sandby-Møller J, Poulsen T, Wulf HC. Epidermal thickness at different body sites: relationship to age, gender, pigmentation, blood content, skin type and smoking habits. Acta Derm Venereol 2003;83:410-413. 